\title{
Abstracts for the Symposium on the Application of Neural Networks to the Earth Sciences
}

\author{
Edited by Donald A. Singer
}

\section{INTRODUCTION}

Artificial neural networks are a group of mathematical methods that attempt to mimic some of the processes in the human mind. Although the foundations for these ideas were laid as early as 1943 (McCulloch and Pitts, 1943), it wasn't until 1986 (Rumelhart and McClelland, 1986; Masters, 1995) that applications to practical problems became possible. It is the acknowledged superiority of the human mind at recognizing patterns that the artificial neural networks are trying to imitate with their interconnected neurons. Interconnections used in the methods that have been developed allow robust learning. Capabilities of neural networks fall into three kinds of applications: (1) function fitting or prediction, (2) noise reduction or pattern recognition, and (3) classification or placing into types.

Because of these capabilities and the powerful abilities of artificial neural networks, there have been increasing applications of these methods in the earth sciences. The abstracts in this document represent excellent samples of the range of applications. Talks associated with the abstracts were presented at the Symposium on the Application of Neural Networks to the Earth Sciences: Seventh International Symposium on Mineral Exploration (ISME-02), held August 20-21, 2002, at NASA Moffett Field, Mountain View, California. This symposium was sponsored by the Mining and Materials Processing Institute of Japan (MMIJ), the US Geological Survey, the Circum-Pacific Council, and NASA. The ISME symposia have been held every two years in order to bring together scientists actively working on diverse quantitative methods applied to the earth sciences. Although the title, International Symposium on Mineral Exploration, suggests exclusive focus on mineral exploration, interests and presentations have always been wide-ranging - abstracts presented here are no exception. was held:

It is through the efforts of the Organizing Committee listed below that the symposium

Dr. Ryoichi Kouda, Geological Survey of Japan, roy.kouda@aist.go.jp

Dr. Katsuaki Koike (Kumamoto University), koike@gpo.kumamoto-u.ac.jp

Treasurer: Toshiaki Ueki (Sanyo Technomarine), ueki@ohti.co.jp

Dr. Donald Singer, United States Geological Survey, singer@usgs.gov

Dr. Joseph Coughlan, NASA, jcoughlan@mail.arc.nasa.gov

Secretary: Kathy Linale, klinale@usgs.gov

\section{REFERENCES}

Masters, T., 1995, Advanced algorithms for neural networks: a C++ sourcebook: John Wiley \& Sons, $431 \mathrm{p}$.

McCulloch, W.S., and Pitts, W., 1943, A logical calculus of the ideas immanent in nervous activity: Bulletin of Mathematical Biophysics, v. 5, p. 115-133.

Rumelhart, D.E., McClelland, J.L., 1986, Parallel distributed processing: explorations in the microstructure of cognition; MIT Press, Cambridge, MA, Volume 1: Foundations, 547 p. 
ABSTRACTS 


\title{
Predicting Lateral-Spread Ground Failure in Coastal California Using a Probabilistic Neural Network
}

\author{
Richard L. BERNKNOPF ${ }^{1}$ and Laura B. DINITZ ${ }^{1}$
}

${ }^{1}$ U.S. Geological Survey, 345 Middlefield Road, Menlo Park, California 94025, U.S.A. Email: rbern@usgs.gov

An important barrier to effective regional risk assessment of earthquake damage is predicting which locations and structures will be affected. A probabilistic neural network based on the Bayesian classifier method is used to classify land parcels into one of three expert-determined risk levels for lateral-spread ground failure due to earthquakes. The expert scientist identified the geologic units susceptible to lateral-spread ground failure using physical attributes and processes and assigned them relative susceptibility values. Here we attempt to produce probabilistic risk maps that illustrate the likelihood of earthquake-triggered hazards from the results of both a neural network and a qualitative response regression model. The results from both methods are compared.

Velocity ratio (a surrogate for site amplification), distance to stream (free-face), slope, and average percent sand in 3,836 cells (10,000 meter square) were used to train and test the network. Slopes greater than six percent and geologic materials older than 10,000 years were excluded. Except for average percent sand, each of the variables was transformed by logarithms to reduce effects of skewness. Half of the cells were randomly selected to be used in training the probabilistic neural network and the other half were used for independent testing. Tests were performed with a probabilistic neural network employing a Gaussian kernel and separate sigma weights for each risk level and each variable.

The neural network assigned 73 percent of 1,925 cells used in independent testing to the same class as the expert. Eighty-six percent of the lowest susceptibility class and 89 percent of the moderate susceptibility class cells that were classified by the neural network matched those determined by the expert. For cells identified by the expert as high risk, the neural network identified only 24 percent in the same class as the expert. Because of the high success rate of probabilistic neural networks in related problems, it seems likely that some variables used by the expert to classify the high-risk land parcels were not included in this analysis. 


\title{
Knowledge Recovery for Continent-Scale Metal Exploration by Neural Networks
}

\author{
Laurent BOUGRAIN ${ }^{1}$, Maria GONZALEZ ${ }^{2}$, Vincent BOUCHOT ${ }^{2}$, G. STEIN $^{2}$, \\ Cassard DANIE ${ }^{2}$, and Frédéric ALEXANDRE ${ }^{1}$
}

${ }^{1}$ LORIA,/INRIA, campus scientifique, BP 239, Postal Code: 54506, Vandoeuvre-Les-Nancy

Cedex, France

Email: bougrain@loria.fr

${ }^{2}$ BRGM, Resources Mineral Division, BP 6009, Cedex, France

Data mining performances are heavily dependent on the quality of the database and the efficiency of the applied data exploration routines. The Mineral Resources Division of the BRGM ( Bureau de Recherches Géologiques et Minières) develops continent-scale Geographic Information Systems (GIS), which support metallogenic research. The research involves understanding of the formation of gold, copper, silver and other deposits and assists in the exploration for new deposits. Application of data mining techniques to such information systems may thus contribute to the discovery of new mineral deposits. Of the different datasets available in the information systems, 25 attribute have been put forward by geological expertise as known factors and potential factors controlling the formation of a gold deposit in the Andes mountain chain (a region with extensive mineral deposits). The attributes include: position (longitude, latitude, altitude) of the deposit, the type and age of the country rock hosting the deposit, the proximity of the deposit to a fault zone distinguished by its orientation in map view, density and focal depth of earthquakes immediately below the deposit, proximity of active volcanoes, geometry of the subduction zone occurring along the westcoast of South America and associated to the Andes mountain chain (e.g. distance between the deposit and the subduction zone, angle of the subduction zone, and distance to the subduction trench). Two labels are applied to the corpus: 'deposits' and 'non-deposits'. A 'deposit" label for a specific metal means that this resource has been extracted at this location. The set of 'non-deposit' labels consists of arbitrary points selected from areas without a reported deposits and with minimal chance to host an undiscovered deposit. 378 examples of 'deposits' and 288 examples of 'non-deposits' were available. Artificial neural networks constitute good tools for certain types of computational modelling (being potentially efficient, easy to adapt and fast). We applied various multilayer perceptrons with 25 inputs, one hidden layer and 2 output units using a softmax function to predict the posteriori probability that a new site belongs to the 'deposit' and 'non deposit' classes. We obtained between 80 and 85 percent correct classification for the gold deposits. Subsequently, as artificial neural networks (such as dynamical and modular networks) can be used to construct a revised model for knowledge extraction we applied the optimal brain damage algorithm by LeCun to order the 25 proposed attributes by their relevance to the classification. The approach demonstrated how neural networks can be used efficiently in a practical problem of mineral exploration, where the general domain knowledge alone is insufficient to satisfactorily model the potential controls on deposit formation using the available information in the continent-scale information system. 


\title{
Use of Fuzzy Membership Input Layers to Combine Subjective Geological Knowledge and Empirical Data in a Neural Network Method for Mineral Potential Mapping.
}

\author{
Warick BROWN ${ }^{1}$, David GROVES ${ }^{1}$, and Tamás GEDEON ${ }^{2}$ \\ ${ }^{1}$ Centre of Global Metallogeny, Department of Geology \& Geophysics, University of \\ Western Australia, Nedlands, WA 6907, Australia \\ Email:wbrown@geol.uwa.edu.au \\ ${ }^{2}$ Department of Information Technology, Murdoch University, Murdoch, WA, Australia
}

Using GIS layers, in which the cell values represent fuzzy membership values, is an effective method of combining subjective geological knowledge with empirical data in a neural network approach to mineral prospectivity mapping. In this study, multilayer perceptron (MLP), probabilistic (PNN) and self-organising map (SOM) neural networks were used to combine up to 17 regional exploration data sets to predict the potential for orogenic gold deposits in the Archaean Kalgoorlie Terrane, Western Australia, in the form of prospectivity maps. Two types of fuzzy membership layers were used. In the first type of layer, the statistical relationships between known gold deposits and rock types in a solid geology GIS layer and a layer showing the nearest type of lithological boundary for each cell were used to convert layers containing categorical data to fuzzy membership values. Layers representing favourable lithologies and favourable lithological boundaries were prepared in this way. Rock units and contact types were ranked according to the number of known deposits associated with each class. Subjective judgement was used to decide which categories are definitely favourable and therefore assigned a membership degree of one. A triangular fuzzy membership function was used to assign fuzzy memberships to all the other rock types (and contact types). The use of fuzzy membership rather than categorical inputs is useful for MLP networks, particularly if there are a large number of classes in the categorical layer. Categorical data are normally converted to numerical form using 1-of-n coding and this leads to a large number of additional input connections, which in combination with small training data set sizes could lead to over-fitting of the training data and poor generalization.

Rheological contrast at lithological boundaries was modeled using a second type of fuzzy membership layer, in which the assignment of fuzzy membership value, although based on geological field observations, was purely subjective. On a regional scale, most large gold deposits in the Kalgoorlie area are sited in structurally isolated, thick competent units or at contacts between layers of contrasting competency, which are oriented between NNW and NNE. The reason for this is that the more competent units tend to fail selectively in this orientation and hence become sites of increased structural permiability and fluid focusing. Subjective estimates of the relative competency of host rocks were based on the degree of silica content, modal mineralogy, grain size, fabric development, texture, and metamorphic grade. For example, banded iron formation and basalt were ranked as very competent whereas ultramafic schists were ranked as very incompetent. All rock types were assigned a ranking, which was used to create a GIS layer containing cell values corresponding to the competency contrast at the nearest lithological boundary. The approach used here could potentially be applied to a large range of subjective data (e.g., favourability of tectonic environment, host stratigraphy, or reactivation along major faults) currently used in regional exploration programs but which would normally not be employed in an empirical neural network approach. 


\title{
Use of Noise to Augment Training Data to Compensate for Lack of Deposit Examples in Training a Neural Network for Mineral Potential Mapping
}

\author{
Warick BROWN $^{1}$, Tàmas GEDEON ${ }^{2}$, and David GROVES ${ }^{1}$ \\ ${ }^{1}$ Centre of Global Metallogeny, Department of Geology \& Geophysics, University of \\ Western Australia, Nedlands, WA 6907, Australia \\ Email:wbrown@geol.uwa.edu.au \\ ${ }^{2}$ Department of Information Technology, Murdoch University, Murdoch, WA, Australia
}

An important problem in applying neural networks to predict the potential for mineral deposits from exploration data is the rarity of deposit training patterns compared to the very large number of patterns that correspond to barren cells. In order to ensure the network correctly learns the patterns in the data, the training data should sample the entire range of variation in the data population. It is also important to adequately represent feature vectors that are located close to decision boundaries in multidimensional feature space. Both these requirements are difficult to fulfil because there are so few deposit examples relative to barren patterns.

The work described here is based on a regional GIS data set, which is being used to examine the potential for orogenic lode-gold deposits in an approximately 100 by 100 kilometre area of the Archaean Yilgarn Block, near Kalgoorlie in Western Australia. MultiLayer Perceptron (MLP) neural networks were trained to predict the presence or absence of deposits. Ten GIS layers in raster format were used to create feature vectors for each 100 metre cell on the map grid. The data were split into training, training-test and test sets. The training set was used to train the network weights, the training-test set was used to determine when to stop training in order to avoid over-fitting the data, and an independent test data set was used to check the performance of the trained network. Since the GIS data was captured at a regional scale, only the 120 deposits with a total contained gold resource $>1000 \mathrm{~kg}$ were used to train and test the neural networks. An approximately equal number of barren patterns were selected randomly from the map area and allocated randomly to the training data sets. The training, training-test and test sets contain 99, 88 and 81 patterns, respectively. Each set contains approximately equal numbers of deposit and barren patterns.

Random noise was added to each component of the original deposit feature vectors to create 22 new patterns from each original pattern. Matching numbers of additional barren patterns were randomly selected from the map grid, resulting in new training and training-test set sizes of 2111 and 1792, respectively. No changes were made to the test data set. Ten networks with different initial weights were trained for each experiment. The same random weight initializations were repeated for separate experiments so that it was clear that any differences in the results were due to changes in the training data and not the starting weights. Experiments using an expanded training set and both expanded training and training-test data sets were performed. Different amounts of uniform and normallydistributed random noise were also tested (i.e., $\pm 5, \pm 10 \pm 20 \pm 40 \& \pm 80 \%$, where each value represents the maximum amount added or subtracted).

Results of the experiments were assessed using both the classification performance of the trained network on the test set as well as statistical measures of the quality of the 9-class prospectivity maps. The latter category comprised Spearman's correlation coefficient (to 
test the correlation between increasing prospectivity map class and the probability that the region corresponding to the highest prospectivity class contains a known deposit), the area under a receiver operating characteristic curve (ROC) curve, and two forms of a capture efficiency ratio. The capture efficiency ratios, D/A and D_D/A, measure the degree to which the most prospective map class accounts for the known deposits. D refers to the percentage of known deposits and A refers to the percentage of the total map area represented by the region with the highest prospectivity class, respectively.

Networks trained using the noise-augmented data sets resulted in significantly increased classification accuracy for the test set (overall) and test-set deposit patterns, area under the ROC curve and capture efficiency (D/A) and D_D/A statistics. The best results were obtained using an MLP network with a 10-20-1 topology and $\pm 40 \%$ noise. With the latter parameters, the following results were obtained; test-set overall percentage correct (67.9, $72.8,76.5)$, test-set deposit patterns $(68.0,77.1,85.7)$, Spearman's correlation coefficient $(0.97,0.99,1.00), \mathrm{D} / \mathrm{A}(8.2,17.9,14.4)$, D_D/A $(105,226,216)$, and area under the ROC curve $(0.79,0.87,0.90)$. The numbers in brackets refer experiments using the original training set, expanded training set and expanded training and training-test data sets, respectively.

The method described here could be applied to increase the number of patterns available for training in other applications, in which training data are rare, difficult or expensive to obtain. 


\title{
Development of a CNN For Better Understanding the Seasonal Responses of a Glacial-Filled Aquifer to Variable Climate and Pumping Conditions
}

\author{
Emery COPPOLA ${ }^{1}$, Mary POULTON ${ }^{2}$, Ferenc SZIDAROVSZKY ${ }^{2}$, and Emmanuel \\ CHARLES ${ }^{1}$
}

${ }^{1} \mathrm{NOAH}, \mathrm{LLC}$

${ }^{2}$ Mining and Geological Engineering, University of Arizona, Tucson, AZ 85721, U.S.A. Email: mpoulton@u.arizona.edu

A computational neural network $(\mathrm{CNN})$ was constructed and trained to estimate monthly groundwater levels in a glacial-sediment aquifer in response to variable pumping and climate conditions. The groundwater system, located within the Towaco Valley Aquifer of northern New Jersey, supplies Montville Township with approximately 2 million gallons of water each day. Recent drought conditions and increasing groundwater pumping of the three production wells have resulted in declining groundwater levels within the 4 square mile watershed, prompting concerns of aquifer overdraft. In order to help assess the long-term sustainability of the aquifer, the developed CNN was used to conduct a sensitivity analysis on the possible effects of pumping and climate conditions on groundwater levels. While a limited number of data (36 records) were available, the resulting training and validation errors were only 5 and 12 percent, respectively. More importantly, the results of the sensitivity analysis agree with the seasonal dynamics of the hydrologic system, as well as the locations of the pumping and observation wells relative to each other. As a result, the Township will be installing continuous water-level recorders so that a CNN utilizing daily inputs and outputs (rather than monthly) can be developed. This CNN should provide greater insights into both short and long-termed influences on groundwater levels. In addition, the CNN may ultimately be used to help manage pumping rates so that potential adverse aquifer impacts can be minimized to the extent practicable. 


\title{
Hybrid Neural Networks for Human-Centered Earth Science Information Processing
}

\author{
Amulya K. GARGA ${ }^{1}$, Eileen S. ROTTHOFF ${ }^{2}$, Timothy S SHAW ${ }^{3}$ \\ ${ }^{1}$ Penn State Applied Research Laboratory, 3075 Research Drive, State College, PA 16801, \\ U.S.A. \\ Email: garga@psu.edu \\ ${ }^{2}$ Penn State Applied Research Laboratory, P. O. Box 30, State College, PA 16804, U.S.A. \\ Email: esr10@psu.edu \\ ${ }^{3}$ Penn State Applied Research Laboratory, 3075 Research Drive, State College, PA 16801, \\ U.S.A. \\ Email: tshaw@psu.edu
}

Extensive research has focused on the development of techniques for multi-sensor data fusion systems in many domains. The increasing amount and complexity of the earth science data collected by NASA remote sensors underscores the need for research into strategies and techniques to facilitate its analysis and understanding. In this paper, an application of artificial neural networks to human-centered earth science information processing is described. This work contributes to improved understanding of multi-source remote sensor data by utilizing earth science domain knowledge, remote sensor data, and other information sources for improved contextual interpretation and understanding. This hybrid approach uses non-commensurate information, explicit domain knowledge and knowledge implicit in the data, to model, represent, and display complex multi-dimensional terrestrial and atmospheric data and processes. Domain knowledge is very general and is usually expressed in terms of rules. Customization of a rule base is extremely tedious and can only be done offline. To illustrate this hybrid method, multi-valued logic rules were developed with three observable parameters that have an impact on forest fires. The input and output variables were allowed to take values of Low, Medium, or High. The rule base consisting of 27 rules were developed for this application. A neural network with 3 input neurons, 4 hidden neurons, and 1 output neuron was trained using the backpropagation training method in MATLAB Neural Network toolbox. The network is able to perform $100 \%$ correct prediction on the rule base. Presently, the neural network is being evaluated with observed data and will be retrained on those. The results of the processing can then be readily visualized with immersive, multi-modal (visual and aural) human-computer interfaces. We believe that human-centered fusion methods will provide aids to reduce cognitive biases, improve the understanding of heterogeneous, multi-source data, and provide increased opportunities for data discovery. 


\title{
Embedding Watershed Geomorphology into Artificial Neural Networks for Direct Runoff Computations
}

\author{
Bin ZHANG ${ }^{1}$ and Rao S. GOVINDARAJU ${ }^{1}$ \\ ${ }^{1}$ School of Civil Engineering, Purdue University, West Lafayette, IN 47907, U.S.A. \\ Email: govind@ecn.purdue.edu
}

We study the problem of estimating direct runoff over a watershed resulting from rainfall excess. The goal of this study is to develop an artificial neural network (ANN) that explicitly accounts for the geomorphologic characteristics of the watershed within its architecture. Such a geomorphology-based artificial neural network (GANN) is utilized to estimate runoff hydrographs from several storms over two Indiana watersheds. The architecture of the GANNs as well as a part of the network connection strengths are determined by watershed geomorphology leading to improved ANN modeling ability. Our results indicate that the GANN model performs more accurately than geomorphologic unit hydrograph (GIUH) model when compared to observed hydrographs. We show that GANNs represent an improvement over conventional ANN models and they appear to hold promise as a tool for estimating watershed runoff. 


\title{
A Fuzzy Neural Network Approach to Predicting Numbers of Undiscovered Mineral Deposits \\ Darryn HEDGER ${ }^{1}$, and Roussos DIMITRAKOPOULOS ${ }^{1}$
}

\author{
${ }^{1}$ W.H. Bryan Mining Geology Research Centre, University Of Queensland, Brisbane QLD \\ 4072, Australia \\ Email: brc@uq.edu.au
}

\begin{abstract}
Mineral exploration is an economically risky business, particularly due to the inherent uncertainties associated with the discovery of mineral resources. Crucial to any exploration risk analysis is a quantitative mineral resource assessment that predicts a region's undiscovered deposit numbers. The assessment must deal with uncertainties in geological data while providing a model describing the uncertainty associated with any prediction of undiscovered deposit numbers. The probabilistic neural network (PNN) is an intrinsic classifier that used a weighted distance function. It can be trained, with data for known deposit classes, to generate nonparametric probability density functions (pdf) describing the probability that unknown data belongs to the respective deposit classes. Deposit numbers can then be modelled from the pdf using confidence limits if the assumption that the training classes are exhaustive and mutually exclusive is true. In mineral resource assessment training classes rarely represent all the possible alternatives and in some case they can overlap making this assumption invalid.
\end{abstract}

This paper demonstrates a hybrid method based upon a probabilistic neural net and fuzzy nearest neighbor (FNN) as a way to measure the uncertainty in the predicted undiscovered deposit numbers. The FNN is an objective fuzzy classifier that uses membership proximity between desired deposit classes, in the unknown data, and known deposit classes in the training data. The resulting classes are fuzzy events, in this case the possibility of a deposit class. Fuzzy probabilities are calculated using class probabilities from the PNN and the fuzzy events from the FNN. This calculation is done via a-level Borel type measures of significance that a deposit class is possible. The fuzzy probabilities represent a fuzzy set or possibility distribution describing the fuzzy probability of a possible deposit class. Estimates of undiscovered deposit numbers based on fuzzy deposit probabilities are not bound by the laws of exhaustiveness and mutually exclusivity, unlike the stand-alone probabilistic based estimates. A case study illustrates how the use of fuzzy probabilities can enhance estimating number of deposits by adding a level of significance to any prediction; for example a probable prediction may become "highly" probable. 


\title{
Analyzing Multi-Hazard Risk for Populations and Infrastructure of the Pacific Rim
}

\author{
David G. HOWELL ${ }^{1}$, Eric S. YURKOVICH ${ }^{2}$, and Heather V. GOSS ${ }^{3}$ \\ ${ }^{1}$ U.S. Geological Survey, 345 Middlefield Rd. MS 975, Menlo Park, CA 94025, U.S.A. \\ Email: dhowell@usgs.gov \\ ${ }^{2}$ U.S. Geological Survey, 345 Middlefield Rd. MS 975, Menlo Park, CA 94025, U.S.A. \\ Email: eyurkovich@usgs.gov \\ ${ }^{3}$ U.S. Geological Survey, 345 Middlefield Rd. MS 975, Menlo Park, CA 94025, U.S.A. \\ Email: hgoss@usgs.gov
}

An exploding population and unprecedented urban development within the last century helped fuel an increase in the severity of natural disasters. The world also has become a more interconnected social structure, as people, information and commodities travel greater distances and service larger populations and cities. This consequence of natural disasters with interrelatedness requires an expanded analysis for understanding the rising risk. To improve disaster planning we propose a model comprising 1) a comparative index of risk to assess the impact of five natural hazards: earthquake, flood, tropical storm, tsunami and volcanic eruptions, 2) population density, 3) a proxy representing the distribution of societal infrastructure, 4) a vulnerability indicator for the elements at risk ( $2 \& 3)$; and 5) a linkage index portraying the connected nature of a 'globalized' world. We depict this relationship with the equation$$
\text { Risk }=\mathrm{f}(\text { Hazard }, \text { Elements at Risk, Vulnerability , Interconnectivity })
$$

Because population and infrastructure are distributed differently, two contrasting representations of risk emerge from this study. Each assessment-accessible to planners and implemented by public-domain data and GIS software-reflects different repercussions from natural disasters: immense losses, either to life or economic activity.
} 


\title{
The Optimum Mining Plan for Phosphorus Adjustment in a Limestone Quarry Using a Genetic Algorithm
}

\author{
Toshihide ITO $^{1}$ and Takashi NISHIYAMA ${ }^{2}$ \\ ${ }^{1}$ Kansai University, Dept. of Informatics, 2-1-1 Ryozenji, 569-1095. Japan \\ Email: toshi@ipcku.kansai-u.ac.jp \\ ${ }^{2}$ Kyoto University, Sakyo-ku, Kyoto, 606-8501, Japan \\ Email: nishiyama@energy.kyoto-u.ac.jp
}

The limestone quarries where slaked lime is produced for steel makers are strongly requested to reduce concentration of phosphorus in their products. In many quarries in Japan, limestone blocks with low concentrations are blended with limestone blocks with high concentrations to keep below the limit of permitted phosphorus quality. The life of the quarry is extended as long as possible by this blending. Some quarries have a geological database to keep accurate records of operations for the phosphorus adjustment.

However, the combination problem of these blocks typically is addressed by Dynamic Programming, that is, all combinations of possible limestone blocks are examined in the solving process and the combination numbers are so huge that the optimum combination could not be solved in practical process times. Though it took less than 20 hours to solve 600 blocks in $2 \mathrm{GHz}$ high performance personal computers, it would take more than 53 days to approximately solve for 1200 blocks. Therefore, the Genetic Algorithm was tried to solve the optimum combination. Firstly, the blocks that can be dug out are chosen in quarry and their digging sequence makes genotype. The Order-base or the Grefenstette method was applied to avoid generating lethal genes in crossover. The fitness value was estimated by the number of products that include less than permitted concentration of phosphorus.

The GA process took only 8 hours to solve for 1200 blocks. Since the processing time is almost proportional to the block numbers, this GA method is practical in large quarries. Moreover, this method is easy to apply to other conditions in mining plans, such as environmental protection, noise prevention and eyesore problems. These problems are becoming important factors in quarries near towns in Japan. 


\title{
Classification of Geographic Images by a Shift Invariant Neural Network
}

\author{
Izumi KAMIYA ${ }^{1}$ \\ ${ }^{1}$ Geographical Survey Institute, Kitasato 1, Tsukuba, Ibaraki, 305-0811, Japan \\ Email: kamiya@gis.go.jp
}

Shift invariant neural networks, a kind of feedforward neural network with error backpropagation learning, are used to classify aerial photographs and other geographic images. Neurons in a layer of the shift invariant neural network are arranged as a gird in 3dimensional space. Two axes, $x$ and $y$, correspond to the spatial axes of the image. The remaining axis, $\mathrm{z}$, corresponds to kinds of neurons in a layer like ordinary feedforward neural networks. The z-axis corresponds to the spectral bands in the input layer and the classification categories in the output layer, and is expected to correspond to appropriate characteristics of the image in the intermediate layers. Classification result of a pixel shall be influenced by only its neighbor pixels; so connections between only spatially neighbor pixels are allowed in the shift invariant neural network. The same input shall bring the same classification results, even if their spatial positions differ; so weights of the connections depend only on the kinds of the neurons and the relative coordinate between the neurons, instead of the absolute coordinates. The first condition reduces the number of connections, and the second reduces the number of independent connections.

The shift invariant neural network has two important characteristics: it extracts both spectral and spatial information simultaneously, and it executes the feature extraction step and the classification step in a narrow sense simultaneously. The shift invariant neural network was applied to two kind of image classification. An operator interpreted and classified the images first. The network classified them using half of the operator's classification result as training samples. The classification result of the network was evaluated using the remaining half as verification samples.

The first example is a geological classification into serpentinite and others using a slope gradation map, which is a monochromatic image generated from DEM shows gradient of the earth surface. Serpentinite-distributed areas are easily interpreted on the image, but cannot successfully classified without spatial information. The answer was 97 percent correct in the verification samples. The success shows the shift invariant neural network has ability to extract special image information. The second example is land use classification into housing areas, agricultural land and forests using a color aerial photograph. The answer was 92 percent correct in the verification samples. 


\title{
Characterizing Content Distributions of Minor Elements in a Limestone Mine Using a Feedforward Neural Network
}

\author{
Katsuaki KOIKE ${ }^{1}$, Setsuro MATSUDA ${ }^{2}$, and Ohmi MICHITO ${ }^{1}$ \\ ${ }^{1}$ Department of Civil Engineering, Faculty of Engineering, Kumamoto University, 2-39-1 \\ Kurokami, Kumamoto 860-8555, Japan \\ Email:koike@gpo.kumamoto-u.ac.jp \\ ${ }^{2}$ Graduate School of Science \& Technology, Kumamoto University, 2-39-1 Kurokami, \\ Kumamoto 860-8555, Japan
}

Limestone is relatively abundant as a non-metallic resource in Japan which, otherwise, has few mineral resources. It is chiefly used as a raw material for cement and as a supplement for iron and steel manufacturing. The factors that determine the suitability of limestone for industrial use and its commercial value are contents of impurities such as silicate and phosphorus. From 244 sample points in 18 drillhole sites in a limestone mine, southwest Japan, we collected the data on four impurity elements, $\mathrm{SiO}_{2}, \mathrm{Fe}_{2} \mathrm{O}_{3}, \mathrm{MnO}$, and $\mathrm{P}_{2} \mathrm{O}_{5}$. It is generally difficult to estimate spatial distributions of these contents, because most of the limestone bodies in Japan are located in the accretionary complex lithologies of Paleozoic and Mesozoic ages. Since the spatial correlations of content data were not clearly shown by the variogram analysis, a feedforward neural network was applied to estimate the content distributions The network structure consists of three layers: input, middle, and output. The input layer has nine neurons and the output layer has four neurons. Three neurons in the input layer correspond with $x, y, z$ coordinates of a sample point and the others are rock types such as crystalline and conglomeratic limestones. Four neurons in the output layer correspond with the amounts of $\mathrm{SiO}_{2}, \mathrm{Fe}_{2} \mathrm{O}_{3}, \mathrm{MnO}$, and $\mathrm{P}_{2} \mathrm{O}_{5}$. Numbers of neurons in the middle layer and training data vary with each estimation point to avoid overtraining of the network. We could detect several important characteristics of the content distributions through the network such as a continuity of low content zones along ENE-WSW. The zones were clarified to correspond with a specific biostratigraphic zone. In addition, we compared two spatial distributions by the neural network and a geostatistical method. Capability of the neural network-based method is superior to the geostatistical method as shown from a viewpoint of estimation errors of multivariate data. 


\title{
Cognitive Exploration by Artificial Neural Networks Ryoichi KOUDA $^{1}$ and Donald A. SINGER ${ }^{2}$
}

\author{
${ }^{1}$ Geological Survey of Japan, 1-1, Higashi 1-chrome, Tsukuba Central 7, Tsukuba, Ibaraki, \\ 305-8567, Japan \\ Email: roy.kouda@aist.go.jp \\ ${ }^{2}$ U.S. Geological Survey, 345 Middlefield Road, Menlo Park, California 94025, U.S.A. \\ Email: singer@usgs.gov
}

Artificial Neural Networks have been applied in fields of earth sciences ranging from flood forecasting and reservoir characterization to remote geologic mapping. In mineral resource exploration these networks contribute to recognizing mineralized and barren zones, typing of ore-forming environments, estimating potential for deposits, and so on. In the past, these kinds of recognition were made through deterministic classification on the one hand, or through statistical analysis on the other hand. Often the source data are too complicated to clearly separate groups or make estimates based on the characteristics, the structure, and the values of weights. Artificial Neural Networks provide a solution to recognize the complicated features of nature and contribute to make the exploration targets clearer and comprehensive through the cognitive processes of the input, reaction and output layer simulations of the brain functions. We present some examples of applications of the use of Artificial Neural Networks in the exploration for kuroko ore deposits. We review some simple programming codes and results of steepest decent, mean square error, perceptron, ADALINE, maximum likelihood, back propagation, and so on. A few related methods of Artificial Neural Networks that could be applied to some earth science targets are also considered. 


\title{
Spatial Modeling Principal Metal Contents of Kuroko Ores and Detecting Influence Factors on Their Distribution by a Feedforward Neural Network
}

\author{
Setsuro MATSUDA ${ }^{1}$, Katsuaki KOIKE ${ }^{2}$, and Toru SUZUKI ${ }^{3}$ \\ ${ }^{1}$ Graduate School of Science \& Technology, Kumamoto University, 2-39-1 Kurokami, \\ Kumamoto 860-8555, Japan \\ ${ }^{2}$ Department of Civil Engineering, Faculty of Engineering, Kumamoto University, 2-39-1 \\ Kurokami, Kumamoto 860-8555, Japan \\ Email: koike@gpo.kumamoto-u.ac.jp \\ ${ }^{3}$ Metal Mining Agency of Japan, 1-24-14 Toranomon, Minato-ku, Tokyo 105-0001, Japan
}

Geostatistics have been widely used for regionalized geologic data having spatial correlation structures. In some cases, these structures cannot be clarified by a semivariogram due to limited data and/or complexity of the data distribution. We applied a feedforward neural network to this kind of problem by choosing chemical content data in a kuroko-deposits area in northern Japan as a case study. Kuroko is the black ore that mainly contains sphalerite, galena, and barite. Kuroko-type deposits are one of the kinds of massive sulfide deposits having genetic relations to submarine volcanic activity. The content data of multiple chemical components were collected from 1917 sample points at 143 drillhole sites. The network structure consists of three layers: input, middle, and output. Input data of the network are the coordinates of sample point, Bouguer anomaly, and rock code. Contents of principal metals of kuroko, $\mathrm{Cu}, \mathrm{Pb}$, and $\mathrm{Zn}$, and value of alteration index are estimated through the network. Numbers of neurons in the middle layer and training data vary with the location to avoid overtraining of the network. Suitable numbers of neurons were determined by repeating cross-validation at each point. Three-dimensional distributions of $\mathrm{Cu}, \mathrm{Pb}$, and $\mathrm{Zn}$ were successfully estimated by the proposed method, as shown by anomaly zones localized near the known kuroko-type deposits and the margins of Tertiary volcanoes or calderas. Using the weights connecting adjacent neurons, sensitivity analysis of the neural network was carried out. Influence factors were calculated in this analysis to clarify geologic factors that affect the distributions of $\mathrm{Cu}, \mathrm{Pb}$, and $\mathrm{Zn}$ contents. Depth and the mudstones in the lower Onnagawa or Nishikurosawa geologic stage were found to be significant factors. Another interesting feature is that some anomaly zones extend along the large sensitivity vectors. 


\title{
Artificial Neural Networks for Mineral Potential Mapping
}

\author{
Alok PORWAL ${ }^{1}$, Emmanuel John CARRANZA ${ }^{2}$, Martin HALE ${ }^{3}$ \\ ${ }^{1}$ International Institute for Geo-information Science and Earth Observation (ITC), Henge- \\ losestraat 99, P.O. Box 6, 7500 AA Enschede, The Netherlands \\ Email: porwal@itc.nl \\ ${ }^{2}$ International Institute for Geo-information Science and Earth Observation (ITC) Henge- \\ losestraat 99, PO Box 6. 7500 AA Enschede, The Netherlands \\ Email: carranza@itc.nl \\ ${ }^{3}$ International Institute for Geo-information Science and Earth Observation (ITC) Henge- \\ losestraat 99, PO Box 6, 7500 AA Enschede, The Netherlands \\ Email: hale@itc.nl
}

A GIS-based application of artificial neural networks (ANN) to map potential for mineral deposits is developed and demonstrated in the south-central part of the Aravalli metallogenic province (western India). Recognition criteria for mineral deposits were represented as multi-class evidential maps, which were subsequently tested for conditional independence vis-à-vis the locations of mineral deposits. The conditionally-independent maps were combined to generate a unique condition (or feature vector) map. The locations of known mineral deposits were divided into two subsets, viz., the training and the validation points. Feature vectors spatially associated with the training points were extracted along with several randomly-selected feature vectors previously modelled as not spatially associated with the training points to generate the training vector subset. Radial basis function neural networks (RBFNN) were used in a more generalised form called radial basis functional link nets (RBFLN) for training the network. This feedforward network has a simple three-layered structure comprising an input layer, the hidden neurodes (radial basis functions or the RBFs) and an output layer. The training was accomplished in two stages: 1) initialisation of the centres, spread parameters and weights; and 2) adjustment of the weights and parameters to minimise total sum-squared error (TSSE). After training the network to recognise the presence or absence of mineral deposits, it was tested and then used for classifying the feature vector map. The output for each feature vector is in the form of a value ranging from 0 (absence of mineral deposit) to 1 (presence of mineral deposit). These output values were used to map zones with favourability for mineral deposits. The threshold favourability values were determined and used to generate a rank favourability map for mineral deposits. Spatial coincidence between the predicted high-favourability areas and the validation points demonstrates the usefulness of the method. 


\title{
Optimization of a Neural Network Model Using a Genetic Algorithm: Predicting Salinity Intrusion in the San Francisco Bay Estuary
}

\author{
T. RAJKUMAR ${ }^{1}$, and David E. THOMPSON ${ }^{2}$ \\ ${ }^{1}$ SAIC, NASA Ames Research Center, M.S. 269-1, Moffett Field, California 94035, U.S.A. \\ Email: rajkumar@mail.arc.nasa.gov \\ ${ }^{2}$ NASA Ames Research Center, M.S. 269-1, Moffett Field, California 94035, U.S.A.
}

The atmosphere, the watershed and the coastal ocean all contain a rich spectrum of spatial and temporal structure, much of which is incorporated into the variability of the San Francisco Bay/Delta. The amount of water accumulated in the Sierra Nevada is the major contributor to the fresh water feeding to the Bay/Delta. Like all estuaries, the S.F. Bay/ Delta is linked to the coastal ocean and to the inland rivers, resulting in high variability at many scales. Also the estuary has undergone extensive human development over the past 150 years, as has its upstream watershed. In particular long-term changes in estuarine conditions provides a more complete picture of the estuary and its ever-changing climatic context. To understand the complex interactions between climatic conditions, hydrologic changes, and tidal effects, over the long term, requires substantial amount of computer models in various fields. We addressed this complex model using a neural network using a Levenberg-Marquardt learning algorithm. We used a genetic algorithm (GA) to optimize the minimum number of training data as well number of hidden neurons to provide faster convergence. The inputs for neural network range from precipitation, snowfall, flow and salinity data collected from monitoring instruments in and around the Bay/Delta. The USGS has established a network of flow monitoring stations in the Delta. Using flow monitoring data from Rio Vista, Three Mile Slough, Jersey Point, and Dutch Slough, a direct estimate of Net Delta Outflow (NDO) is computed. NDO is an arithmetic summation of river inflows, precipitation, agricultural consumptive demand, and project exports. NDO characterizes the upstream watershed. Apart from hydrodynamic data, the stations collect physical and water quality data on a continuous basis monitored one meter below the surface. The parameters provided from the monitoring stations are water temperature, potential hydrogen, dissolved oxygen, air temperature, electrical conductivity [hence, salinity], wind speed, wind direction, solar radiation intensity, and chlorophyll. The GA optimized neural network is trained to understand hydrodynamics, runoff and water quality of the Bay/Delta. This hybrid model is a good predictor for any variable in the Bay/Delta and it helps to understand the complexity very rapidly. 


\title{
Use of a Probabilistic Neural Network to Reduce Costs of Selecting Construction Rock
}

\author{
Donald A. SINGER ${ }^{1}$ and James D. BLISS ${ }^{2}$ \\ ${ }^{1}$ U.S. Geological Survey, 345 Middlefield Road, Menlo Park, California 94025, U.S.A. \\ Email: singer@usgs.gov \\ ${ }^{2}$ U.S. Geological Survey, DeConcini Building, 520 North Park Ave., Room 355, Tucson, \\ AZ 85719-5035, U.S.A. \\ Email: jbliss@usgs.gov
}

Rocks used as aggregate in construction in temperate climates deteriorate to varying degrees due to repeated freezing and thawing. The magnitude of the deterioration depends on the rock's properties. Aggregate, including crushed carbonate rock, is required to have minimum geotechnical qualities before it can be used in asphalt and concrete construction. In order to reduce chances of premature and expensive repairs, extensive freeze-thaw tests are conduced on potential construction rocks. These tests typically involve 300 freeze-thaw cycles and can take four to five months to complete. Less time consuming tests that (1) predict durability as well as the extended freeze-thaw test or that (2) reduce the number of rocks subject to the extended test, could save considerable amounts of money. Here we use a probabilistic neural network to try and predict durability as determined by the freeze-thaw test using four rock properties measured on 843 limestone samples from the Kansas Department of Transportation.

Modified freeze-thaw tests, which take about two weeks, and less time consuming specific gravity-dry, specific gravity saturated, and modified absorption tests were conducted on each sample. Durability factors of 95 or more as determined from the extensive freeze-thaw test are viewed as acceptable_rocks with values below 95 are rejected. If only the modified freeze-thaw test is used to predict which rocks are acceptable, about 45 percent are misclassified. When 421 randomly selected samples and all four standardized and scaled variables were used to train a probabilistic neural network, the rate of misclassification of 422 independent validation samples dropped to 28 percent. The network was trained so that each class (group) and each variable had its own coefficient (sigma). In an attempt to further reduce errors, an additional class was added to the training data to predict durability values greater than 84 and less than 98, resulting in only 11 percent of the samples being misclassified. About 43 percent of the test data was classed by the neural net into the middle group - these rocks should be subject to full freeze-thaw tests. Thus, use of the probabilistic neural network would mean that the extended test would only need be applied to 43 percent of the samples, and 11 percent of the rocks classed as acceptable would fail early. About 6 percent of the test samples were consistently predicted to meet standards and yet failed the extended freeze-thaw test-these samples should be examined for possible errors in the extended tests or for clues to identify a new variable to properly class them. 


\title{
Typing Mineral Deposits Using Their Grades and Tonnages in an Artificial Neural Network
}

\author{
Donald A. SINGER ${ }^{1}$ and Ryoichi KOUDA ${ }^{2}$ \\ ${ }^{1}$ U.S. Geological Survey, 345 Middlefield Road, Menlo Park, California 94025, U.S.A. \\ Email: singer@usgs.gov \\ ${ }^{2}$ Geological Survey of Japan, 1-1, Higashi 1-chrome, Tsukuba Central 7, Tsukuba, Ibaraki, \\ 305-8567, Japan \\ Email: roy.kouda@aist.go.jp
}

\begin{abstract}
A test of the ability of a probabilistic neural network to classify deposits into types on the basis of deposit tonnage and average $\mathrm{Cu}, \mathrm{Mo}, \mathrm{Ag}, \mathrm{Au}, \mathrm{Zn}$, and $\mathrm{Pb}$ grades is conducted here. The purpose is to examine whether this kind of system might serve as a basis for integrating geoscience information available in large mineral databases to classify sites by deposit type. Benefits of proper classification of many sites in large regions are relatively rapid identification of terranes permissive for deposit types and recognition that specific sites might be worth exploring further.
\end{abstract}

Ore tonnages and average grades of 1,137 well-explored deposits from published grade and tonnage models representing 13 deposit types were used to train and test the network. Tonnages were transformed by logarithms and grades by square roots to reduce effects of skewness. All values were scaled by subtracting the variable's mean and dividing by its standard deviation. Half of the deposits were randomly selected to be used in training the probabilistic neural network and the other half were used for independent testing. Tests were performed with a probabilistic neural network employing a Gaussian kernel and separate sigma weights for each class (type) and each variable (grade or tonnage).

Deposit types were selected to challenge the neural network. For many types, tonnages or average grades are significantly different from other types, but individual deposits may plot in the grade and tonnage space of more than one type. Porphyry $\mathrm{Cu}$, porphyry $\mathrm{Cu}-\mathrm{Au}$, and porphyry $\mathrm{Cu}-\mathrm{Mo}$ types have similar tonnages and relatively small differences in grades. Redbed $\mathrm{Cu}$ deposits typically have tonnages that could be confused with porphyry $\mathrm{Cu}$ deposits, also contain $\mathrm{Cu}$ and, in some cases, $\mathrm{Ag}$. Cyprus and kuroko massive sulfide types have about the same tonnages, $\mathrm{Cu}, \mathrm{Zn}, \mathrm{Ag}$, and $\mathrm{Au}$ grades. Polymetallic vein, sedimentary exhalative $\mathrm{Zn}-\mathrm{Pb}$, and $\mathrm{Zn}-\mathrm{Pb}$ skarn types contain many of the same metals. Sedimenthosted $\mathrm{Au}$, Comstock $\mathrm{Au}-\mathrm{Ag}$, and low-sulfide Au-quartz vein types are principally $\mathrm{Au}$ deposits with varying amounts of Ag.

Among the largest classification errors are skarn $\mathrm{Zn}-\mathrm{Pb}$ and Cyprus massive sulfide deposits classed by the neural network as kuroko massive sulfides-24 and 63 percent error respectively. Other large errors are the classification of 92 percent of porphyry $\mathrm{Cu}-\mathrm{Mo}$ as porphyry $\mathrm{Cu}$ deposits. The number of deposits used to train the neural network was 25 or less for each of these deposit types with large errors, suggesting that some errors might be due to using too few training samples. Given our intent to test the neural network under the most difficult conditions, an overall 75 percent agreement between the experts and the neural network is considered excellent. Over 91 percent of the gold deposit types were properly classed and 98 percent of porphyry $\mathrm{Cu}$ deposits were classes as some type of porphyry $\mathrm{Cu}$ deposit. The overall success rate could probably be raised to more than 90 percent with the addition of information on the presence of a few rock types. 


\title{
Comparison of Ordinary Kriging and a Neural Network for Interpolation of Two Dimensional Data
}

\author{
Jorge Kazuo YAMAMOTO' ${ }^{1}$, Katsuaki KOIKE ${ }^{2}$, and Setsuro MATSUDA ${ }^{3}$ \\ ${ }^{1}$ Department of Environmental and Sedimentary Geology, University of Sao Paulo, Brazil, \\ Rua do Lago, 562, CEP 05508-900 - São Paulo - SP, Brazil \\ Email: jkyamamo@usp.br \\ ${ }^{2}$ Faculty of Engineering, Kumamoto University, Japan \\ ${ }^{3}$ Graduate School of Science \& Technology, Kumamoto University, 2-39-1 Kurokami, \\ Kumamoto 860-8555, Japan
}

This paper discusses some characteristic features of ordinary kriging and a neural network as local methods for interpolation of two-dimensional data. Bias and smoothness derived from both methods are presented and discussed in this paper. Ordinary kriging estimation is based on an underlying spatial correlation function (semivariogram), previously modeled from data points. This method provides estimates with minimum error variance, which ensures local accuracy, but not global accuracy that is the reproduction of the spatial correlation function. On the other hand, an artificial neural network (ANN) is a network of many simple units, which are connected and passing signals to each other. ANN can be used for function approximation, classification, or interpolation. The connections are made by weights, which are adjusted for a training pattern and so successively for each training pattern to minimize an error term so that at the end of this training process, the adjusted weights can be used for prediction.

In this paper we compare performance of both ordinary kriging and an artificial neural network for data interpolation in two-dimensional space. The cross-validation procedure was chosen to carry out this comparative study, because for each data point we have the actual value and the estimated one. Moreover, the cross-validation was based on varying the size of the local neighborhoods, i.e., 4, 8 and 12 nearest neighbor samples were taken for estimating each data point. A multi-layer neural network with one hidden layer (5 neurons) was used in this study. Maximum total error was established to be equal to 0.001. As long as the local neighborhood increases the number of steps increases, in order to ensure convergence to the maximum error. Thus, we used a varying maximum number of steps according to local neighborhood (100000 steps for 4 points, 300000 steps for 8 points and 600000 points for 12 points). Synthetic data were considered for this study (mean equal to 2.65 and standard deviation equal to 3.376). This data set presents a positive asymmetric distribution, probably lognormal distribution because its high coefficient of variation (1.274). Therefore, this data set simulates distributions of rare metals in mineral deposits, such as gold, tungsten, etc. Analyzing results of this comparative study we can conclude that RMS errors are slightly smaller for ordinary kriging (1.547, 1.576 and 1.658, respectively for 4,8 and 12 nearest points) than for neural network interpolation $(1.721,1.939$ and 2.066 , for the same sequence of nearest points). Displaying results on scattergrams (actual values on $\mathrm{Y}$ axis and estimated values on $\mathrm{X}$ axis) we observe that the slopes of the least squares lines are less than one $(0.996,0.774$ and 0.757$)$ for neural network predicted values; and systematically greater than one $(1.058,1.186$ and 1.217$)$ for ordinary kriging estimates. In addition, correlation coefficients are slightly higher for ordinary kriging estimates $(0.891,0.893$ and 0.882$)$ than for the neural network predictions $(0.859,0.838$ and 0.806). However, in terms of rank correlation, for 8 and 12 points, neural network predictions present higher values $(0.923$ and 0.914$)$ than ordinary kriging estimates $(0.915$ and 0.904). Ordinary kriging estimates are more susceptible to the smoothing effect than 
the neural network estimates. This means that the variance of ordinary kriging estimates is always less than the variance of the actual values. For neural network predictions, the variance of predicted values using 4 nearest points is less than the variance of the actual values, showing that the neural network interpolation based on a small neighborhood produces some smoothing effect. However, for increasing neighborhood sizes, the variances of neural network predicted values are greater than the variance of the actual values. Finally comparing interpolated images with actual images, we conclude that all ordinary kriging images are smoothed, while neural network images (for 8 and 12 points) present a good spatial correlation with the actual image. 


\title{
Application of a Fuzzy Neural Network Based on Chaos Theory in Dam Safety Monitoring
}

\author{
XU Hongzhong ${ }^{1}$, WU Zhongru ${ }^{2,}$ and SHI Bin ${ }^{1}$ \\ ${ }^{1}$ Department of Earth Sciences, Nanjing Univ., Nanjing 210093, China \\ Email:xhzh@elong.com \\ ${ }^{2}$ College of Water Conservancy \& Hydropower Engineering, Hohai University, Nanjing \\ 210098, China
}

Dams have social and economical impact on the environment, and their safety and performance are of great importance. Dam monitoring produces a large quantity of data. The analysis of monitoring data is an important factor when determining the behavior of a dam. Mathematical models such as a statistical model, or a deterministic model are widely used in dam safety monitoring field in China. These models are based on linear regression, which cannot reflect the nonliearity in the dam observation data. Combining chaos theory with neural network theory, a fuzzy network model of phase space for analyzing the monitoring data on dams is established in the paper. The analysis on the horizontal displacements of a gravity dam is given as an example of the application of the model.

First, the raw monitoring data is adjusted to remove the deterministic components (i.e. the trends effect, the seasonal effect, and the effect of hydrostatic load on the dam) in the data by using regression analysis. Second, according to chaos theory, the phase space construction of a filtered time series is performed. The chaotic invariants of filtered time series such as the correlation dimension, the Lyapunov exponent, are calculated. The results show that the data observation data is a chaotic time series. Then, a Fuzzy neural network model of phase space is developed based on the filtered data. Chaos theory is used to determine the inputs of the fuzzy neural network, and the architecture of the fuzzy neural network is determined. The model is used to predict horizontal displacement. The results indicate that the prediction based on this model outperforms the statistical model. 